\title{
ON THE RADIAL AND NONTANGENTIAL MAXIMAL FUNCTIONS FOR THE DISC
}

\author{
RICHARD L. WHEEDEN ${ }^{1}$
}

\begin{abstract}
Positive powers of the radial and nontangential maximal functions of a function which is harmonic or analytic in the unit disc are shown to have equivalent integrals with respect to Borel measures satisfying the growth condition $\mu(2 I) \leqq c \mu(I)$ for every interval $I$.
\end{abstract}

Let $u(z)$ be a function which is harmonic or analytic in the unit disc $D=\left\{z: z=r e^{i x}, 0 \leqq r<1,-\pi<x \leqq \pi\right\}$. For $0<\alpha<1$, let $\Gamma_{\alpha}(x)$ denote the open subset of $D$ bounded by the two tangent lines from $e^{i x}$ to the circle $|z|=\alpha$ and the longer of the two arcs of $|z|=\alpha$ between the points of tangency. Let

$$
N_{0}(u)(x)=\sup _{0 \leqq r<1}\left|u\left(r e^{i x}\right)\right| \quad \text { and } \quad N_{\alpha}(u)(x)=\sup _{z \in \Gamma_{\alpha}(x)}|u(z)|, \quad 0<\alpha<1 \text {, }
$$

denote the radial and nontangential maximal functions of $u$.

Let $\mu$ be a nonnegative, periodic, finite Borel measure on $|z|=1$. If $I$ is an interval (arc) on $|z|=1$ and $a>0$, let $a I$ denote the interval concentric with $I$ whose length is $a$ times that of $I$. We assume that $\mu$ satisfies

$$
\mu(2 I) \leqq c \mu(I)
$$

for every interval $I$, where $c$ is a positive constant independent of $I$.

A related condition, introduced in an equivalent form by $\mathrm{C}$. Fefferman, is that there exist positive constants $c$ and $\varepsilon$ such that for every interval $I$ and every Lebesgue measurable subset $E$ of $I$,

$$
\mu(E) / \mu(I) \leqq c(|E| /|I|)^{\varepsilon},
$$

where $|E|$ denotes the Lebesgue measure of $E$. Such a measure is absolutely continuous with respect to Lebesgue measure, and a known argument (see Remark (d), §1, of [5]) shows it satisfies (1). On the other hand, by a result of C. Fefferman and B. Muckenhoupt [3], there are measures which satisfy (1) but not (2). Our result here is that powers of $N_{0}(u)$ and $N_{\alpha}(u)$ have equivalent integrals with respect to measures $\mu$ satisfying (1).

Received by the editors March 29, 1973 and, in revised form, May 25, 1973.

AMS (MOS) subject classifications (1970). Primary 30A78, 31A99.

${ }^{2}$ Supported in part by NSF Grant GP 38540. 
THEOREM. If $u(z)$ is harmonic or analytic in $D, \mu$ satisfies $(1), 0<p<\infty$ and $0<\alpha<1$ then

$$
\int_{-\pi}^{\pi}\left\{N_{\alpha}(u)(x)\right\}^{p} d \mu(x) \leqq c \int_{-\pi}^{\pi}\left\{N_{0}(u)(x)\right\}^{p} d \mu(x)
$$

with $c$ independent of $u$.

In case $d \mu(x)=d x$, this result is contained in Corollary 2, p. 170 of [4]. The proof given there can be easily adapted to the general case for measures satisfying (2). For such $\mu$, the finiteness of the integral on the left in the Theorem is equivalent to the statement that $u$ belongs to a weighted version of the classical Hardy space $H^{p}$ (see $\S 1$ of [5]). To prove the Theorem for a measure which only satisfies (1), we will need a different argument, based on observations of $D$. Burkholder and $R$. Gundy [1].

For $z \in D$ and $0<\delta<1-|z|, B(z, \delta)$ will denote the subset $\{\zeta:|z-\zeta|<\delta\}$ of $D$. Different positive constants will be denoted by the same $c$ followed by the parameters on which they depend.

LeMma 1. Let $u(z)$ be a continuous function defined for $z \in D$ and let $0<\alpha<\beta<1$. If $\mu$ satisfies (1) there is a constant $c_{1}=c_{1}(\alpha, \beta, \mu)$ so that for all $y>0$

$$
\mu\left\{x: N_{\beta}(u)(x)>y\right\} \leqq c_{1} \mu\left\{x: N_{\alpha}(u)(x)>y\right\} .
$$

For other forms of this lemma, see Lemma 2 of [2] and Lemma 1 of [5].

Proof. Choose a positive number $t=t(\alpha, \beta)$ so small that for any interval $I$ on $|z|=1$ with $|I| \leqq t$,

$$
D-\bigcup_{x \notin I} \Gamma_{\alpha}(x) \subset\{(\beta+1) / 2<|z|<1\} .
$$

Fix $y>0$ and write the open set $\left\{N_{\alpha}(u)>y\right\}$ as the union of nonoverlapping intervals $I_{j}$. If $\left|I_{j}\right|>t$ for any $j$, then by (1) there is a constant $c_{1}=c_{1}(t, \mu)$ such that $\mu\left(I_{j}\right) \geqq c_{1} \mu(-\pi, \pi)$. Therefore, $\mu\left\{N_{\alpha}(u)>y\right\} \geqq c_{1} \mu\left\{N_{\beta}(u)>y\right\}$, and Lemma 1 is proved. If all $I_{j}$ have $\left|I_{j}\right| \leqq t$, then since

$$
\{z:|u(z)|>y\} \subset \bigcup_{j}\left(D-\bigcup_{x \notin I_{j}} \Gamma_{\alpha}(x)\right),
$$

a simple geometric argument shows that

$$
\left\{N_{\beta}(u)>y\right\} \subset \bigcup_{j} a I_{j},
$$

where $a$ is a constant larger than 1 which depends on $\alpha$ and $\beta$ but not on $j$. 
Hence

$$
\begin{aligned}
\mu\left\{N_{\beta}(u)>y\right\} \leqq \sum_{j} \mu\left(a I_{j}\right) & \leqq c_{1} \sum_{j} \mu\left(I_{j}\right) \quad(\text { by }(1)) \\
& =c_{1} \mu\left\{N_{\alpha}(u)>y\right\} .
\end{aligned}
$$

LEMMA 2. Let $u(z)$ be a bounded function which is harmonic or analytic in $D$ and let $0<\alpha<\beta<1$ and $k>1$. If $\mu$ satisfies (1) there is a constant $c_{2}=c_{2}(\alpha, \beta, k, \mu)$ such that for all $y>0$

$$
\mu\left\{x: N_{\alpha}(u)(x)>y, N_{\beta}(u)(x)<k N_{\alpha}(u)(x)\right\} \leqq c_{2} \mu\left\{x: N_{0}(u)(x)>y / 4\right\} .
$$

This lemma is an adaptation of an argument given in [1].

Proof. With each $x$ associate $a$ point $z \in \Gamma_{\alpha}(x)$ such that $|u(z)|>$ $\frac{1}{2} N_{\alpha}(u)(x)$. Fix $y>0$ and let $S=\left\{N_{\alpha}(u)>y, N_{\beta}(u)<k N_{\alpha}(u)\right\}$. We first claim that there is a positive constant $\varepsilon_{0}=\varepsilon_{0}(\alpha, \beta, k)$ such that $|u(\zeta)|>$ ${ }_{4}^{1} N_{\alpha}(u)(x)$ for all $x \in S$ and $\zeta \in B\left(z, \varepsilon_{0} \delta\right), \delta=1-|z|$. To see this, fix $x \in S$ and let $a=|u(z)|$, so that $N_{\beta}(u)(x)<2 k a$. Since $z \in \Gamma_{\alpha}(x)$ and $\beta>\alpha$, there is by the geometry of the situation a positive number $s=s(\alpha, \beta)$, independent of $x$ and $z \in \Gamma_{\alpha}(x)$, such that $B(z, s \delta)$ lies in $\Gamma_{\beta}(x)$. In particular, $|u(\zeta)|<2 k a$ if $\zeta \in B(z, s \delta)$. If $\zeta \in B(z, s \delta / 2)$ then $B(\zeta, s \delta / 2) \subset B(z, s \delta)$, and therefore, since by $[7$, p. 275], there is an absolute constant $c$ so that

we have

$$
|\nabla u(\zeta)| \leqq \frac{c}{s \delta}\left(|B(\zeta, s \delta / 2)|^{-1} \iint_{B(\zeta . s \delta / 2)}|u(\tau)|^{2} d \tau\right)^{1 / 2},
$$

$$
|\nabla u(\zeta)| \leqq c(2 k a) / s \delta, \quad \zeta \in B(z, s \delta / 2) .
$$

Choose $\varepsilon_{0}$ satisfying $0<\varepsilon_{0}<\operatorname{Min}[s / 2, s / 8 c k]$. If $\zeta \in B\left(z, \varepsilon_{0} \delta\right)$, we obtain from the last inequality and the mean-value theorem applied to $u$ between $z$ and $\zeta$ (or applied separately to the real and imaginary parts of $u$ if $u$ is analytic) that

$$
\begin{aligned}
|u(\zeta)| & \geqq a-2\left(\varepsilon_{0} \delta\right)(c 2 k a / s \delta)=a\left(1-\varepsilon_{0} 4 c k / s\right) \\
& >a / 2>N_{\alpha}(u)(x) / 4 .
\end{aligned}
$$

This proves our claim and, since $x \in S$, that $|u(\zeta)|>y / 4$ for $\zeta \in B\left(z, \varepsilon_{0} \delta\right)$. For $x \in S$, let $J(x)$ denote the interval which is the projection of $B\left(z, \varepsilon_{0} \delta\right)$ onto $|z|=1$, and let $l(x)$ denote the smallest interval with center $x$ which contains $J(x)$. By the geometry of the situation,

$$
|J(x)| \geqq c|I(x)|, \quad c=c(\alpha, \beta, k)>0,
$$

and therefore by (1)

$$
\mu(J(x)) \geqq c \mu(I(x)), \quad c=c(\alpha, \beta, k, \mu)>0 .
$$


Moreover, by our claim,

$$
J(x) \subset\left\{x: N_{0}(u)(x)>y / 4\right\} .
$$

The intervals $I(x)$ cover $S$, so by p. 304 of [6] we may select a positive integer $m$ and points $x_{i} \in S, i=1,2, \cdots$, in such a way that $S \subset \bigcup_{i} I\left(x_{i}\right)$, and no point is contained in more than $m$ different $I\left(x_{i}\right)$ 's. Then

$$
\begin{aligned}
\mu(S) & \leqq \sum_{i} \mu\left(I\left(x_{i}\right)\right) \leqq c \sum_{i} \mu\left(J\left(x_{i}\right)\right), \quad c=c(\alpha, \beta, k, \mu) \quad(\text { by }(3)) \\
& \leqq m c \mu\left(\bigcup_{i} J\left(x_{i}\right)\right) \leqq m c \mu\left\{x: N_{0}(u)(x)>y / 4\right\} \quad(\text { by }(4)) .
\end{aligned}
$$

This completes the proof of Lemma 2.

To prove the Theorem, first suppose that $u(z)$ is bounded and harmonic or analytic in $D$. For $0<\alpha<\beta<1$ and $k>1$,

$$
\begin{aligned}
& \mu\left\{N_{\alpha}(u)>y\right\} \\
& \quad \leqq \mu\left\{N_{\alpha}(u)>y, N_{\beta}(u)<k N_{\alpha}(u)\right\}+\mu\left\{N_{\alpha}(u)>y, N_{\beta}(u) \geqq k N_{\alpha}(u)\right\} .
\end{aligned}
$$

Therefore, by Lemma 2 , there exists $c_{2}=c_{2}(\alpha, \beta, k, \mu)$ such that

$$
\begin{aligned}
p \int_{0}^{\infty} y^{p-1} \mu\left\{N_{\alpha}(u)>\right. & y\} d y \\
\leqq & c_{2} p \int_{0}^{\infty} y^{p-1} \mu\left\{N_{0}(u)>y / 4\right\} d y \\
& \quad+p \int_{0}^{\infty} y^{p-1} \mu\left\{N_{\alpha}(u)>y, N_{\beta}(u) \geqq k N_{\alpha}(u)\right\} d y .
\end{aligned}
$$

The last integral equals $\int_{\left\{N_{\beta}(u) \cdot k N_{\alpha}(u)\right\}} N_{\alpha}(u)^{p} d \mu$, which is at most

$$
k^{-p} \int_{-\pi}^{\pi} N_{\beta}(u)^{p} d \mu \leqq c_{1}^{p} k^{-\nu} \int_{-\pi}^{\pi} N_{\alpha}(u)^{p} d \mu, \quad c_{1}=c_{1}(\alpha, \beta, \mu),
$$

by Lemma 1 . Since $u$ is bounded all these integrals are finite. From (5),

$$
\int_{-\pi}^{\pi} N_{\alpha}(u)^{p} d \mu \leqq 4^{p} c_{2} \int_{-\pi}^{\pi} N_{0}(u)^{p} d \mu+c_{1}^{p} k^{-v} \int_{-\pi}^{\pi} N_{\alpha}(u)^{p} d \mu .
$$

The first and last integrals here are the same. Since $c_{1}$ is independent of $k$, we may choose $k$ so large that $c_{1}^{p} k^{-p} \leqq \frac{1}{2}$, and the Theorem follows in this case.

The case of arbitrary harmonic or analytic $u$ can be deduced from the case of bounded $u$ by putting $u_{r}(z)=u(r z), 0<r<1$. Since $u_{r}$ is bounded 
and $N_{0}\left(u_{r}\right)(x) \leqq N_{0}(u)(x)$, we have

$$
\int_{-\pi}^{\pi} N_{\alpha}\left(u_{r}\right)^{p} d \mu \leqq c \int_{-\pi}^{\pi} N_{0}\left(u_{r}\right)^{p} d \mu \leqq c \int_{-\pi}^{\pi} N_{0}(u)^{p} d \mu,
$$

with $c$ independent or $r$ and $u$. The result follows from the monotone convergence theorem by letting $r \rightarrow 1$.

\section{REFERENCES}

1. D. Burkholder and R. Gundy, Boundary behavior of harmonic functions in a half-space and Brownian motion, Ann. Inst. Fourier. 23 (1973).

2. - Distribution function inequalities for the area integral, Studia Math. 44 (1972), 527-544.

3. C. Fefferman and B. Muckenhoupt, Two nonequivalent conditions for weight functions, Proc. Amer. Math. Soc. (to appear).

4. C. Fefferman and E. Stein, $H^{p}$ spaces of several variables, Acta Math. 129 (1972), 137-193.

5. R. Gundy and R. Wheeden, Weighted integral inequalities for the nontangential maximal function, Lusin area integral and Walsh-Paley series, Studia Math. (to appear).

6. $M$. de Guzman, $A$ covering lemma with applications to differentiability of measures and singular integral operators, Studia Math. 34 (1970), 299-317. MR 41 \#8621.

7. E. Stein, Singular integrals and differentiability properties of functions, Princeton Univ. Press, Princeton, N.J., 1970.

Department of Mathematics, University Heights Campus, Rutgers University, New Brunswick, New Jersey 08903 